\title{
NUMERICAL SIMULATION OF ATHEROSCLEROTIC PLAQUE GROWTH USING TWO-WAY FLUID-STRUCTURAL INTERACTION
}

\author{
C. X. $\mathrm{CHEN}^{1}$, Y. DING ${ }^{1}$ and J. A. GEAR ${ }^{\otimes 1}$ \\ (Received 31 May, 2012; revised 5 September, 2012; first published online 15 November 2012)
}

\begin{abstract}
This paper presents a numerical investigation of plaque growth in a diseased artery using the two-way fluid-structural interaction (FSI) technique. An axis-asymmetric $45 \%$ stenosis model is used as the base model to start the plaque growth approximation. The blood is modelled as a non-Newtonian fluid described by the Casson model. The artery tissue is assumed to be a nonlinear material. The two-way FSI simulation is carried out in a way that mimics the unsteady blood flow through a diseased artery by using a pulsatile flow condition. After each flow velocity cycle, the numerical results are extracted and used to modify the stenosis geometry based upon critical wall shear stress (WSS) values and an accepted relationship between the concentration of low density lipoprotein and WSS. The simulation procedure is repeated until the growthupdated stenosis morphology reaches $79 \%$ severity. The behaviour of the flow velocity is analysed at each growth stage, together with the WSS, to determine the change of plaque morphology due to growth. The effects of WSS and pressure on the artery wall at the final stage (79\% severity) of the plaque growth model are also compared with results from the authors' previous work, to demonstrate the importance of the morphology change in plaque growth modelling.
\end{abstract}

2010 Mathematics subject classification: primary 76Z05; secondary 65Z05.

Keywords and phrases: atherosclerosis, stenosis, fluid-structural interaction, plaque morphology, wall shear stress.

\section{Introduction}

The transportation of low density lipoprotein (LDL) enables lipids like cholesterol and triglycerides to be carried within the water-based bloodstream. When LDL accumulates on artery walls, atherosclerotic plaque can start to form. Plaque is an accumulation of white blood cells, especially macrophages that have taken up oxidized LDL. When these cells die, their contents are released, which attracts more

\footnotetext{
${ }^{1}$ School of Mathematical and Geospatial Sciences, RMIT University, Melbourne, Victoria 3001, Australia; e-mail: colin.chen@rmit.edu.au, yan.ding@rmit.edu.au,jag@rmit.edu.au.

(C) Australian Mathematical Society 2012, Serial-fee code 1446-1811/2012 \$16.00
} 
macrophages and creates an extracellular lipid core near the inner surface of the plaque (adjacent to the fluid). Conversely, the outer, older portions of the plaque become more calcific, less metabolically active and more physically stiff over time.

Due to multiple biochemical events and the corresponding biomechanical responses involved, the biological process of plaque growth and rupture has thus far been very difficult to investigate in laboratories. Alternatively, mathematical modelling has been used to study such processes. It has been shown that wall shear stress (WSS) plays a vital role in atherosclerosis [2, 8, 9]. A low WSS location in an artery is closely associated with the phenomenon of high LDL concentration. Using fluorescence microscopy, Naiki and Karino [6] observed the accumulation of macromolecules on the surface of a cultured endothelial cell monolayer. Their observation revealed that the amount of accumulation increased sharply when WSS approached zero. Most recently, Soulis et al. [10] investigated LDL distribution in human arteries by numerical simulation of non-Newtonian blood flow through six image-based human diseased right coronary artery models using the Fluent software package. Their numerical results showed that low WSS was related to high LDL accumulation on the artery wall. An important outcome of their research is the quantitative relationship between LDL accumulation and WSS, revealing a doubling of the normalized LDL accumulation when WSS decreased below a critical value.

Based on the common findings in the literature from both in vitro studies and mathematical/numerical modelling, the current research puts forward the hypothesis that LDL accumulation on an artery wall is the sole contributor to plaque development. Starting from a three-dimensional axis-asymmetric mild $45 \%$ severity stenosis model as the base geometry, this study models plaque growth by utilizing the numerical results obtained from a series of two-way fluid-structural interaction (FSI) simulations. Incorporating the relationship between WSS and LDL accumulation $[6,10]$, the plaque morphology is updated based upon calculated WSS values. The growth-updated model is then used as the new stenosis geometry for the next round of FSI simulation. This procedure repeats until the stenosis severity is increased beyond the $78 \%$ critical level. In this paper, the method of growth modelling is described and the growth-updated plaque morphologies at various stages are presented. Furthermore, the WSS and pressure variations at the final stage of the growth-updated stenosis model with a lipid pool are also presented and compared with the literature. These results show the importance of morphology and structure in the study of plaque growth and collapse.

\section{Methods}

2.1. The base plaque model The plaque growth modelling starts from the three-dimensional axis-asymmetric $45 \%$ stenosis model reported previously by the authors [1] as the base model; see Figure 1. The major features of the base model are summarized in Figure 2. The centre of the coordinates is located at the centre of the plaque base plane on the artery wall. Figure 2(a) illustrates the cross section of 


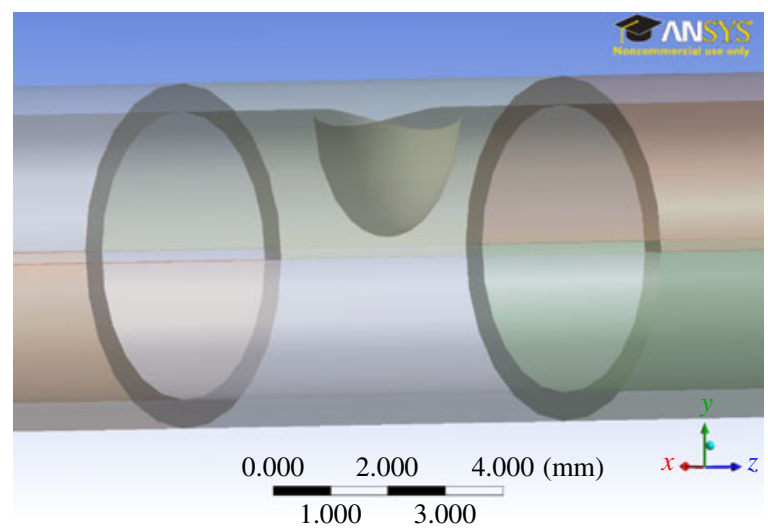

Figure 1. Stenosis shape for the base model.

the base plaque model (solid lines) in the $Z O Y$ plane, and Figure 2(b) that in the $X O Y$ plane.

The sinusoidal pulsatile flow waveform of time period $0.345 \mathrm{~s}$ is superimposed on a steady flow at the inlet as shown in Figure 2(c). This waveform is used so that the modelling results can be validated with the experimental work of Ojha et al. [7] and with the numerical study by Lee et al. [4], in which the same waveform was used. Three cycles are simulated to achieve flow pattern repeatability for all the models. The blood is modelled as a non-Newtonian fluid described by the Casson model [3]. At high shear values, the Casson model behaves like a Newtonian fluid model, while at low shear values, it exhibits non-Newtonian behaviour. These properties are ideal for modelling blood flow, as blood is a non-Newtonian fluid at low shear rates but can exhibit Newtonian behaviour at high shear rates. The artery wall is assumed to be hyper-elastic; its material property is governed by the neo-Hookean model with wall thickness of $1 \mathrm{~mm}$. The stenosis for the base model is assumed to be a semi-elliptical volume, with major axes $A 0$ and $B 0$ and semi-minor axis $Y 0$; see Figure 2(a). To ensure the accuracy of the numerical solution, the stenosis is located $F \mathrm{~mm}$ from the inlet, determined by the condition that a developed flow is present prior to the stenosis. The length of the artery, $L \mathrm{~mm}$, is also long enough that a fully developed flow is achieved prior to the outlet. These dimensions, together with the inner diameter of the artery, $D \mathrm{~mm}$, are given in Table 1 . The meshes for all the models are dominated by hexahedra elements. A mesh independence test was carried out to solve for the fluid convection-diffusion equations and the artery wall deformation, using the two-way FSI method in ANSYS ${ }^{\circledR}$ CFX $^{\mathrm{TM}} /$ Mechanical $^{\mathrm{TM}}$ (Academic Research, Release 13.0) software. Figure 3 presents the mesh independence test results for the base model, showing that WSS is converged when mesh density in the computational domain is increased above 120000 cells. 
(a)

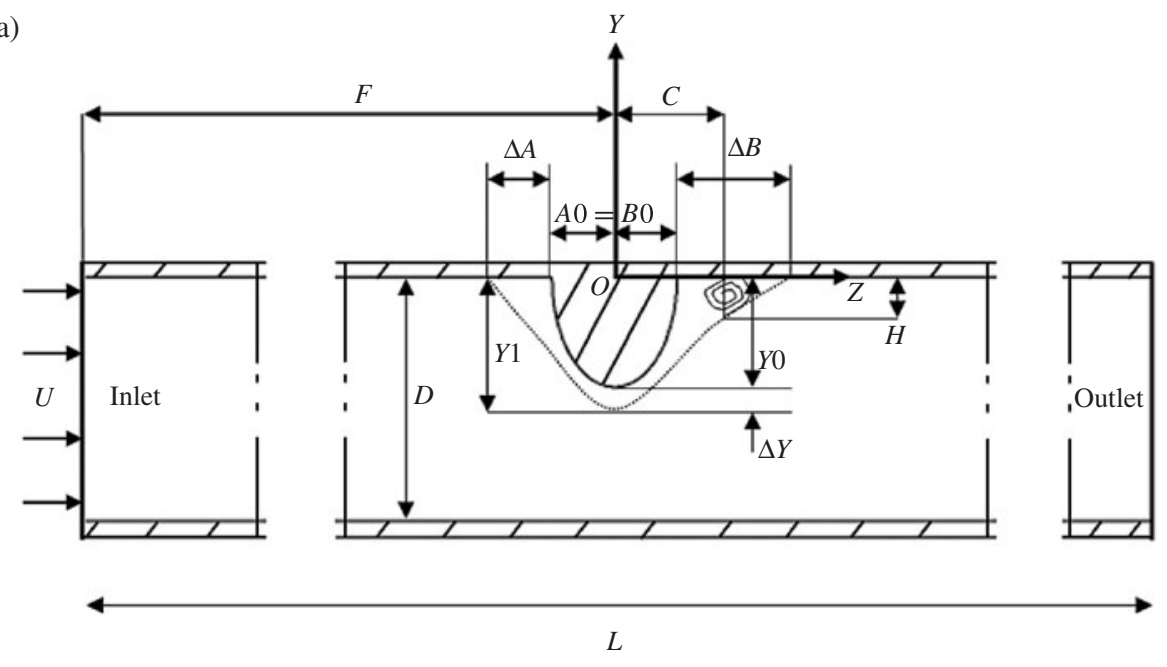

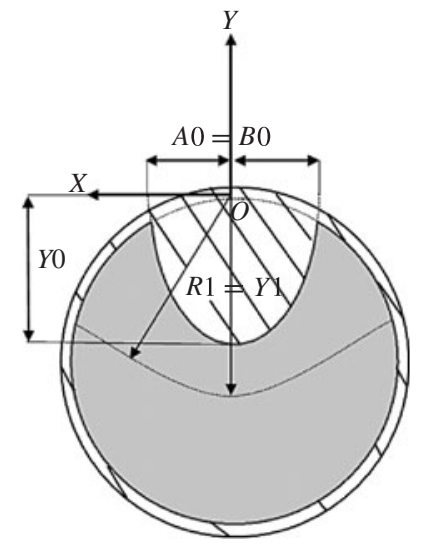

(b)

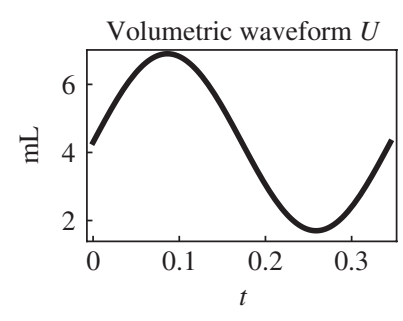

(c)

FigURE 2. Sinusoidal pulsatile waveform specified at the inlet and $Z O Y$ and $X O Y$ views of the base model.

2.2. Computational equations and procedure The two-way FSI method is used to capture the compliance of the artery wall. The fluid solver uses the finite volume method (FVM), while the structural solver adopts the finite element method (FEM). The pressure obtained from the fluid solver at the interface of the fluid and solid domains is then passed, as a load, onto the wall in the structural solver to solve for the deformation of the artery. The displacement obtained in the structural solver is then passed back into the fluid solver to adjust the fluid domain. This procedure continues until the convergence target is reached, that is, the normalized change of interface loads is less than 0.001 between the FVM and FEM solutions.

At each time step $(0.05 \mathrm{~s})$, the blood flow, being viscous and incompressible, is governed by the continuity and Navier-Stokes equations. The governing equations in 


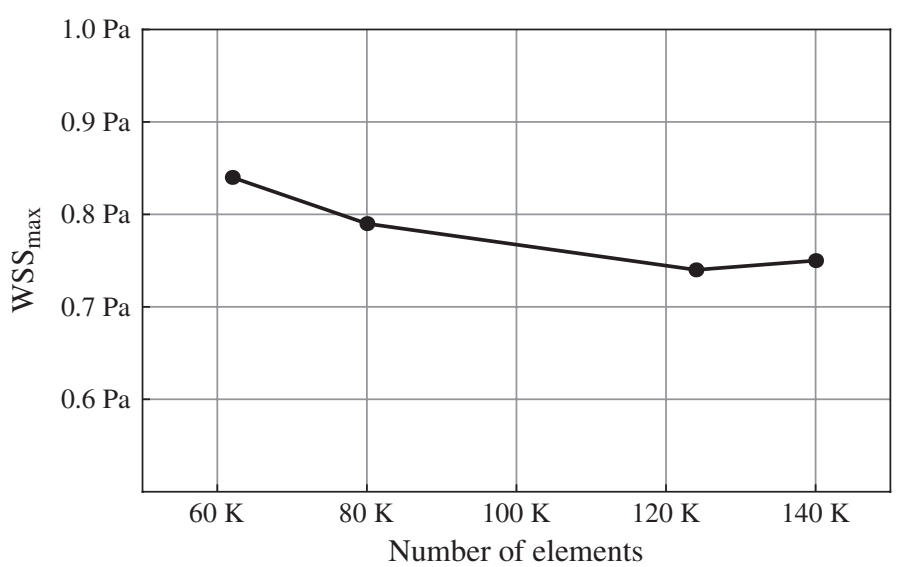

FIGURE 3. Maximum WSS values for four mesh densities.

control volume integral form are

$$
\frac{d}{d t} \int_{V} \rho \mathbf{U}_{\mathbf{i}} d V+\int_{S} \rho_{f} \mathbf{U}_{\mathbf{j}} \mathbf{U}_{\mathbf{i}} d n_{j}=-\int_{S} P d n_{j}+\int_{S} \mu_{f} \frac{\partial \mathbf{U}_{\mathbf{i}}}{\partial \mathbf{X}_{\mathbf{j}}} d n_{j}, \quad \int_{S} \mathbf{U}_{\mathbf{j}} d n_{j}=0,
$$

where $V$ denotes the volume of integration, $S$ denotes the surface of integration, $d n_{j}$ are the differential components of the outward normal vector, $\rho_{f}\left(\mathrm{~kg} \mathrm{~m}^{-3}\right)$ is the density of the fluid (see Table 1), $\mathbf{U}\left(\mathrm{m} \mathrm{s}^{-1}\right)$ is velocity, $P(\mathrm{~Pa})$ is pressure, $t(\mathrm{~s})$ is time, $\mathbf{X}(\mathrm{m})$ is displacement and $\mu_{f}$ (Pas) is viscosity. The Casson model for the viscosity of blood is

$$
\mu_{f}=\left[\left(\eta^{2} J_{2}\right)^{1 / 4}+2^{-1 / 2} \tau_{y}^{1 / 2}\right]^{2} J_{2}^{-1 / 2},
$$

where $J_{2}=(\dot{\gamma} / 2)^{2}$ is a function of shear strain $\dot{\gamma}, \tau_{y}=0.1(0.625 h)^{3}$ is the yield stress, and $\eta=\eta_{0}(1-h)^{-2.5}$ is Casson's viscosity coefficient, where $\eta_{0}=0.0012 \mathrm{~Pa}$ and $h=0.37$ is the volume fraction of red blood cells [3].

The governing equation for the artery is

$$
\rho_{w} \frac{d^{2} d_{i}}{d t^{2}}=\frac{\partial \sigma_{i j}}{\partial x_{j}}
$$

where $\rho_{w}\left(\mathrm{~kg} \mathrm{~m}^{-3}\right)$ is the density of the artery wall (see Table 1$), d_{i}(\mathrm{~m})$ is displacement and $\sigma_{i j}(\mathrm{~Pa})$ is the stress tensor. The strain energy of the solid is governed by the neo-Hookean model:

$$
W=\frac{\mu_{w}}{2} I_{1}-3+\frac{\kappa}{2}(J-1)^{2}
$$

Here $\mu_{w}(\mathrm{MPa})$ represents initial shear modulus (see Table 1), $\kappa(\mathrm{MPa})$ represents bulk modulus, $I_{1}$ is the first deviatoric invariant of the left Cauchy-Green deformation tensor, and $J=\operatorname{det} \mathbf{F}$ where $\mathbf{F}$ is the deformation gradient tensor. 
TABLE 1. Model parameters.

\begin{tabular}{llll}
\hline$L$ & $67.584 \mathrm{~mm}$ & $F$ & $16.292 \mathrm{~mm}$ \\
$D$ & $5 \mathrm{~mm}$ & $A 0, B 0$ & $1.292 \mathrm{~mm}$ \\
$Y 0$ & $2.25 \mathrm{~mm}$ & $v$ (Poisson ratio) & 0.45 \\
$\rho_{f}$ & $1050 \mathrm{~kg} \mathrm{~m}^{-3}$ & $\rho_{w}$ & $1000 \mathrm{~kg} \mathrm{~m}^{-3}$ \\
$\kappa$ (stenosis) & $240 \mathrm{MPa}$ & $\kappa$ (wall) & $60 \mathrm{MPa}$ \\
$\mu_{w}$ (stenosis) & $24 \mathrm{MPa}$ & $\mu_{w}$ (wall) & $6 \mathrm{MPa}$ \\
\hline
\end{tabular}

2.3. Growth modelling The graph presented by Soulis et al. [10, Figure 7] shows a normalized LDL distribution (ratio of surface to fluid concentration) under different WSS values. For normalized LDL concentrations up to 1.02, no surface LDL accumulation occurs. At high WSS values (for example, 2-8 Pa), the maximum normalized LDL concentration reaches 1.1. For lower range WSS values (less than $2 \mathrm{~Pa}$ ), the normalized LDL concentration increases and can be as much as 1.2. Hence, double the surface accumulation is possible at low WSS. Since the focus of this study is on the evolution of the stenosis, it can be inferred that the regions with around zero WSS values promote approximately twice as much LDL accumulation as elsewhere on the artery wall. Since WSS is proportional to the tangential velocity of the flow in the layer adjacent to the artery wall (the velocity gradient adjacent to the artery wall), it is realistic to assume that a stenosis would have the maximum LDL build-up in the post-stenotic region within the flow recirculation zone. In the pre-stenotic region, it is expected that the stagnation zone is much smaller than the flow recirculation zone in the post-stenotic region. The pre-stenotic region is also subjected to the impact of higher fluid velocity during the systolic phase, with the potential of washing away a portion of LDL left behind during the diastolic phase. It is also expected that no stagnation zone would exist at the throat of the stenosis, where fluid velocity is at its highest. Hence the build-up of LDL, in the post-stenotic region according to the relationship of Soulis et al. [10], would be about double the build-up for the throat and pre-stenotic regions.

The first step of the growth modelling is to determine the size of the flow stagnation region in the front and the recirculation zone at the back of the stenosis, as these regions would eventually be filled with high concentrations of LDL, hence forming the new shape of the stenosis. Numerical results of the velocity profile in the vicinity of the stenosis are used to determine the sizes of these regions. The length and depth of these regions then form the new construction points for the updated stenosis geometry. The horizontal growths $\Delta A$ and $\Delta B$ (see Figure 2(a)) are determined by the locations on the wall where the $Z$-component of the fluid velocity in the adjacent layer is $U_{Z}=0$. Since WSS in the post-stenotic region approaches zero, lower than that in the pre-stenotic region, the plaque morphology would show that the growth 


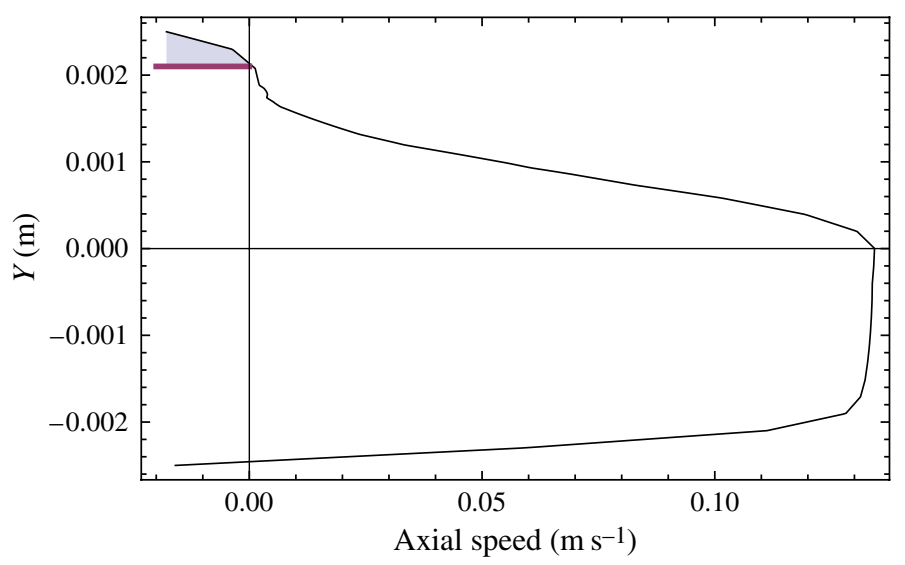

FIgure 4. The $Z$-component velocity profile $U_{Z}$ across the diameter of the artery at the distance $C \mathrm{~mm}$ in the post-stenotic region for the base model, $45 \%$ stenosis severity, diastolic phase. The difference in the $y$-axis of the shaded area defines $H(\mathrm{~mm})$.

in the pre-stenotic region, $\Delta A$, is determined as half of the growth in the post-stenotic region, $\Delta B$, based on the Soulis et al. [10] relationship of the LDL concentration versus WSS and the Naiki-Karino [6] critical around-zero WSS value. The height of the fluid recirculation zone, $H \mathrm{~mm}$, and its distance from the centre plane of the stenosis, $C \mathrm{~mm}$, are determined by the point in the fluid domain where the normal distance from the wall is the maximum in the recirculation zone, when $U_{Z}$ changes its sign from negative to positive, as shown in the shaded area in Figure 4. Since WSS is the highest at the throat of the stenosis, the vertical growth at the throat, $\Delta Y \mathrm{~mm}$ (see Figure 2(a)), is determined as $\Delta Y=\frac{1}{2} H$, that is, a $50 \%$ reduction of the growth in the around-zero WSS region based on the method of growth. The updated geometry is indicated by the dotted curve in Figure 2(a). The point $(C, H)$ is used as a construction point in updating the shape of the plaque geometry. Figure 2(b) illustrates the growth-updated plaque morphology (in the dotted curve) incorporated onto the base model in the XOY plane view. Here $Y 1$ and $R 1$ represent the growth in the vertical and radial directions, respectively. Due to modelling complexity and lack of data in the literature for the growth in the radial direction, we assume the radial growth to be equivalent to the vertical growth. This method of growth is repeated after each FSI simulation until the stenosis severity has reached or gone beyond the $78 \%$ critical level.

\section{Results and discussion}

Figure 5 shows the axial component of the fluid (blood) velocity near the stenosed (solid curve) and opposite (dotted curve) sides of the wall, extracted in the diastolic phase. The shaded area in Figure 5 indicates the stenosis location and the length of the plaque along the artery wall. A $2 \mathrm{~mm}$ negative velocity zone indicating the fluid reversal area is observed in the post-stenotic region. This length is used to form the 


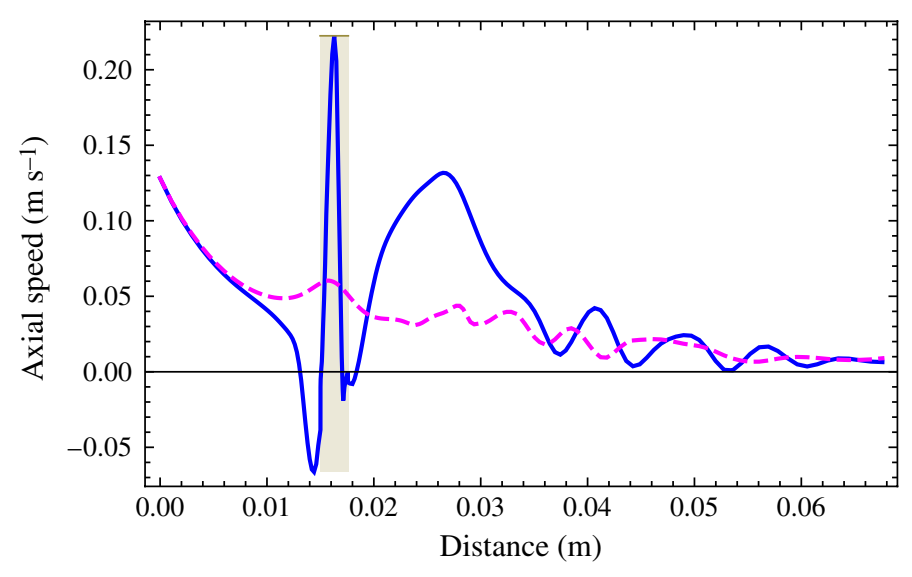

Stenosis side

Opposite wall $=--+-$

FigurE 5. The Z-component velocity profile for the base model, 45\% stenosis severity, diastolic phase. The shaded area indicates the stenosis location and length along the artery.

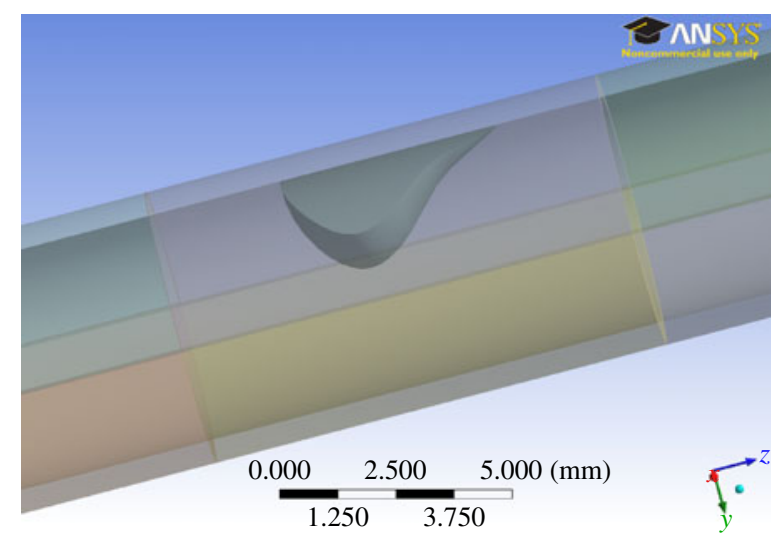

FIGURE 6. Stenosis shape for the shape correction model.

initial post-stenotic tapering of the stenosis as shown in Figure 6, which is now referred to as the shape correction model.

The results of the FSI simulation on the shape correction model show a $6 \mathrm{~mm}$ flow recirculation zone, as the potential plaque growth portion, in the post-stenotic region. However, the results also show a $5 \mathrm{~mm}$ fluid stagnation zone in the pre-stenotic region, which is deemed unrealistic. The expected flow profile for a realistic stenosis growth model should show the pre-stenotic region (high WSS region) having around half as much plaque growth as the post-stenotic region (around-zero WSS region), according to the method of growth detailed in Section 2.3. Thus, the plaque morphology in the 


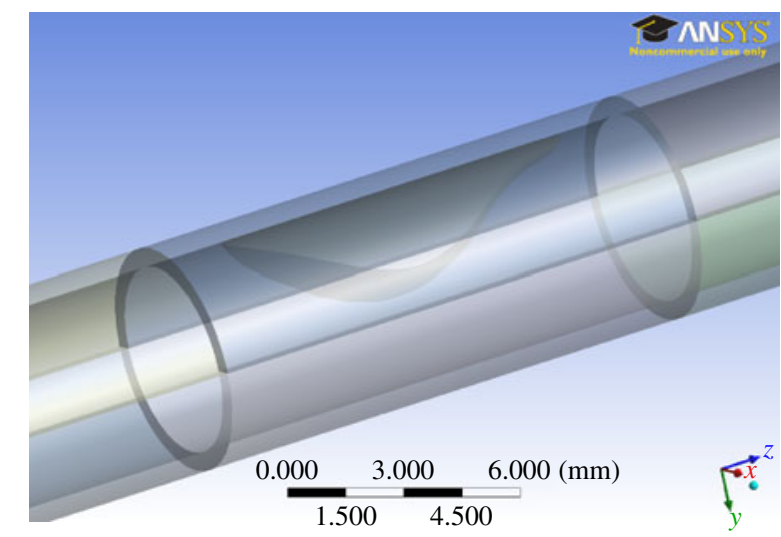

FIGURE 7. Stenosis shape for the 53\% growth-updated stenosis model.

shape correction model is unrealistic due to the fact that the model has no tapering present in the pre-stenotic region, resulting in an unrealistic velocity profile.

Based on the above analysis, the improved shape correction model is constructed with a $6 \mathrm{~mm}$ post-stenotic growth determined from the numerical simulation, and a $3 \mathrm{~mm}$ pre-stenotic growth determined based on the method of growth. Similarly, the vertical growth is also determined. In this case, the height of the fluid recirculation zone is found to be $0.8 \mathrm{~mm}$. Consequently, a $0.4 \mathrm{~mm}$ vertical growth at the throat region is added. The new stenosis is then constructed from these three points (prestenotic, throat and post-stenotic). The improved shape correction model geometry becomes a 53\% stenosis model, as shown in Figure 7 . To check if the plaque morphology of the $53 \%$ stenosis model is realistic, the model is subjected to the FSI simulation. The results show a pre-stenotic fluid stagnation zone of $1 \mathrm{~mm}$ and a poststenotic flow recirculation zone of $2 \mathrm{~mm}$, providing a perfect agreement with the Soulis et al. [10] relationship. This procedure is repeated until the stenosis severity reaches $79 \%$; see Figure 8.

The FSI simulation of the $79 \%$ stenosis model presents some challenges as the mesh in the neighbourhood of the plaque including the plaque is highly distorted, consequently preventing the simulation from being completed. Considering that a $79 \%$ stenosis is developed over a much longer time period than a less severe stenosis, it is reasonable to assume that material properties of the artery wall and plaque fibrous cap should be stiffer than those in the lower severity models as an accumulated calcification effect is expected. Furthermore, clinically a $78 \%$ stenosis artery has been considered as the worst severity case, beyond which the likelihood of life-threatening plaque rupture is imminent, resulting in myocardial infarction. In order to gain insight for the stress-strain field in the artery under such severe conditions, some adjustments for the artery material properties have to be made to complete the simulation through the full pulsatile flow cycle. 


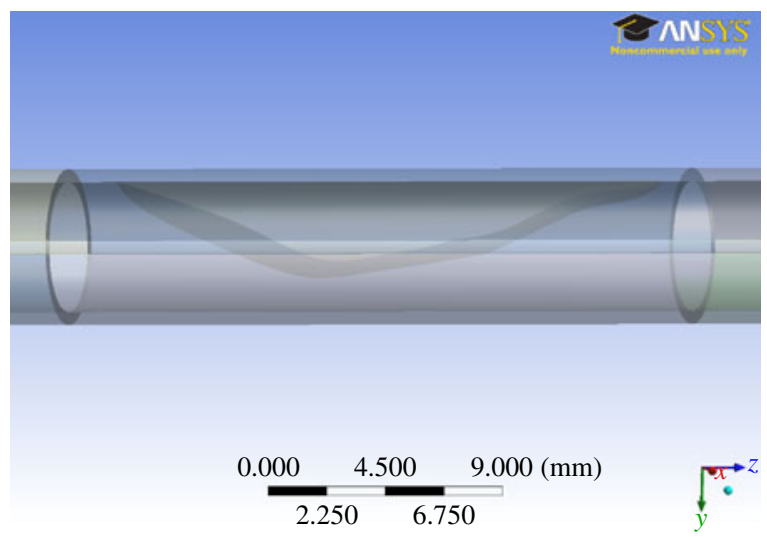

FiGURE 8. Stenosis shape for the $79 \%$ growth-updated stenosis model.

The stiffness of the artery wall is gradually increased to $30 \mathrm{MPa}$ (shear modulus), equivalent to a bulk modulus of $300 \mathrm{MPa}$. The stiffness in the stenosis region is doubled to $48 \mathrm{MPa}$ (shear modulus) and $480 \mathrm{MPa}$ (bulk modulus). This ensures a stable numerical solution over the full flow cycle. Instability at lower wall stiffness suggests the possibility of wall rupture. Although the increase in stiffness is significant, the values are still within the acceptable range. As reported in the literature, $\mathrm{Li}$ and Gillard [5] used bulk moduli of $1600 \mathrm{MPa}$ and $3000 \mathrm{MPa}$ for the wall and fibrous cap, respectively. The FVM results for the fluid provide an insight into the flow behaviour through such an extreme stenosis. The FEM results for the solids contain detailed stress and strain distributions in the artery wall and plaque fibrous cap with varying stiffness, which will be reported in a follow-up research paper.

The axial component of the fluid velocity for the $79 \%$ severity stenosis model is significantly different when compared to the lower severity cases. In Figure 9, the peak magnitude of the velocity component at the centre of the stenosis is equally high for both sides of the wall, with the nondiseased side having slightly higher values for a longer period of time. Several flow recirculation zones are shown in the post-stenotic region of the nondiseased side of the wall, while one deep flow recirculation zone is shown on the diseased side of the wall. The height of the recirculation zone in the poststenotic region also indicates that, if the same growth law is used, the further growth of the stenosis would exceed $100 \%$ stenosis severity, resulting in a complete blockage of the artery. Certainly, this is not possible as fatal plaque rupture would occur before such growth could be achieved.

The final stenosis geometry model reported in this communication is the addition of a lipid pool to the $79 \%$ growth-updated stenosis model. Including the lipid pool region in the $79 \%$ growth-updated stenosis model allows a comparison of results with that of the $78 \%$ uniform stenosis model with lipid pool reported previously [1]. The comparisons of the WSS and pressure profiles between these two types of models 


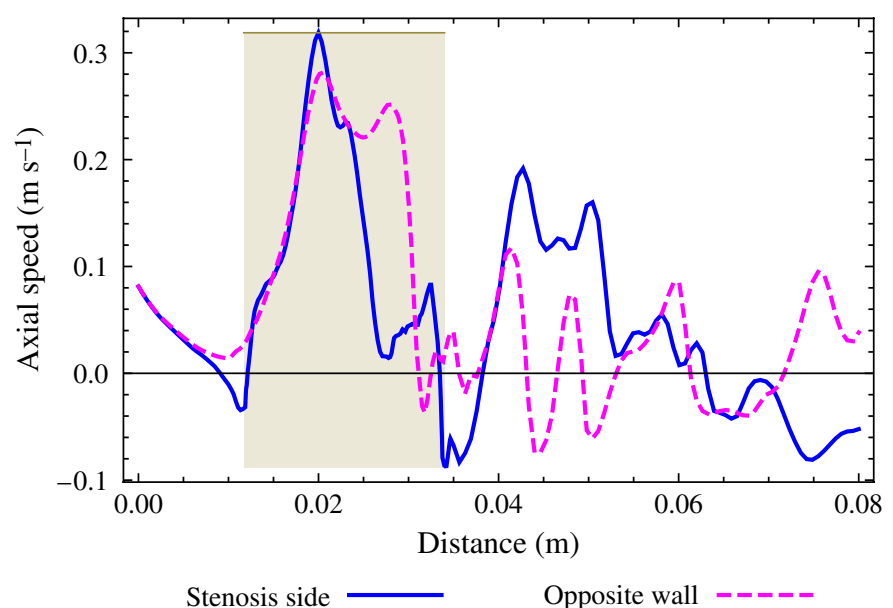

FIGURE 9. The Z-component velocity profile for $79 \%$ growth-updated stenosis model, diastolic phase. The shaded area indicates the stenosis location and length along the artery.

reveal alarming differences in the flow behaviour, particularly in the systolic flow phase. Figure 10 presents WSS variations along the artery wall of the $79 \%$ growthupdated stenosis model including lipid pool at systolic and diastolic phases. The peak WSS value on the stenosis side wall occurs at systolic phase and is about 1.6 Pa. This is significantly lower than that of the $78 \%$ uniform stenosis model with lipid pool, which is $2.5 \mathrm{~Pa}$ (see Figure 11). However, the peak WSS value on the healthy side of the artery wall is about $1.5 \mathrm{~Pa}$ for the $79 \%$ growth-updated stenosis model, while being $0.7 \mathrm{~Pa}$ in the $78 \%$ uniform stenosis model. The WSS values remain high well into the post-stenotic region for the $79 \%$ model, while the $78 \%$ model shows less fluctuation in the post-stenotic region. Since the morphology of the $79 \%$ growth-updated stenosis in the current study is more realistic than that of the $78 \%$ uniform stenosis in our previous communication [1], the WSS and pressure profiles of the $79 \%$ model are more reliable if used for plaque collapse analysis.

The method of growth modelling proposed in this paper enables a systematic approach in utilizing the numerical flow data to construct the new stenosis model after each FSI simulation. The growth-updated plaque geometries reported in this paper show more realistic features than those reported previously [1], demonstrating that the method is a good approximation for studying the formation of atherosclerosis.

It is noted that the time period $(0.345 \mathrm{~s})$ used in this study is too small compared to a human heart rate of approximately $1 \mathrm{~Hz}$. In the next stage of this research, a physiological pulsatile waveform of $1 \mathrm{~Hz}$ at the inlet will be used for modelling of plaque growth. The result will be compared to that presented in this paper.

The FSI simulation results also provide a valuable insight into the stress-strain field of the artery and plaque which is otherwise difficult to obtain in the laboratory. Due to the lack of data in the literature, the assumptions made for the radial plaque growth 

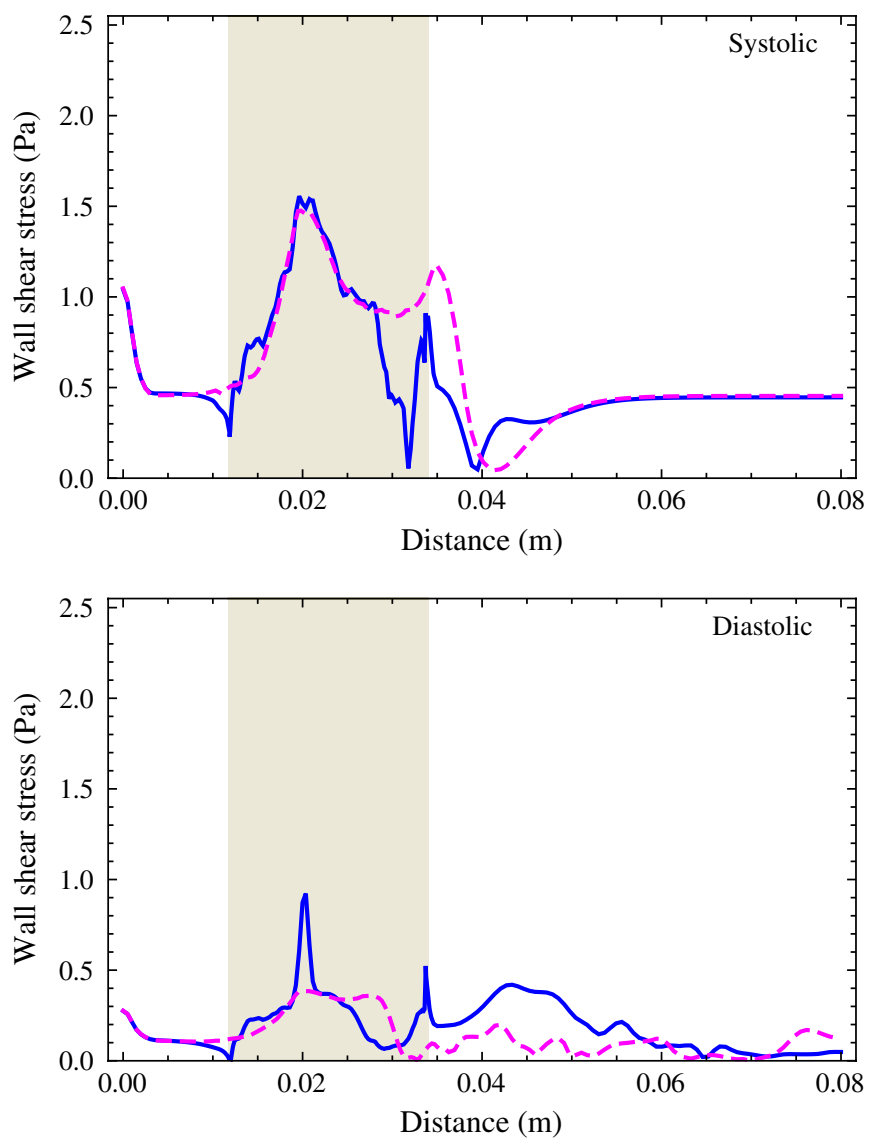

Stenosis side

Opposite wall - - - -

FIGURE 10. WSS distributions for the $79 \%$ growth-updated stenosis model with lipid pool, systolic and diastolic phases. The shaded area indicates the stenosis location and length along the artery.

may affect the accuracy of the simulation results. Furthermore, the accuracy in the plaque growth modelling would be improved if a multi-phase flow condition were implemented in the FSI simulation procedure. This is not considered in the current study due to the modelling complexity involved and a lack of computational resources.

\section{Conclusion}

In conclusion, by utilizing the around-zero WSS value observed by Naiki and Karino [6] and the quantitative relationship of LDL concentration versus WSS reported by Soulis et al. [10], the current study proposes a method of plaque growth and successfully produces more realistic plaque morphologies as the growth of the stenosis progresses to various severity stages. The FSI simulation results demonstrate the 

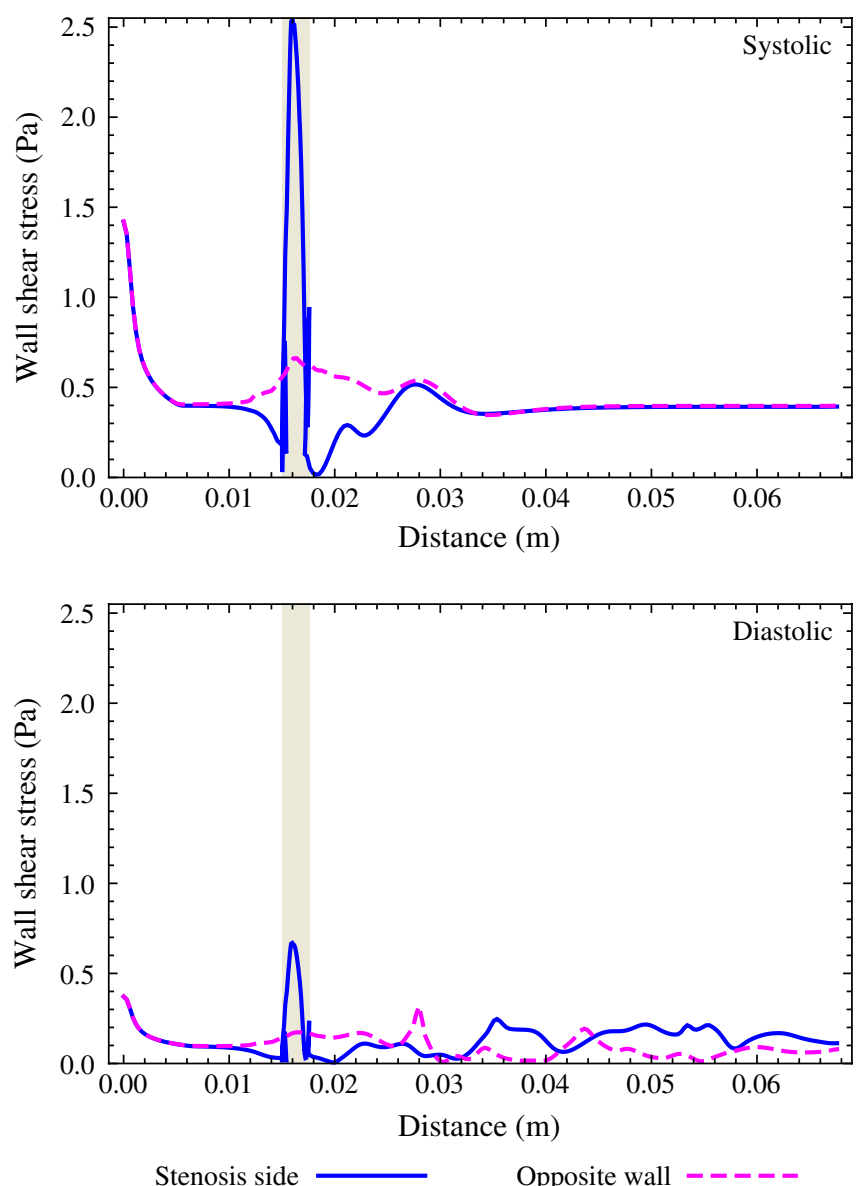

FIGURE 11. WSS for the 78\% uniform stenosis model with lipid pool, systolic and diastolic phases. The shaded area indicates the stenosis location and length along the artery.

importance of the morphology change in plaque growth modelling. A growthupdated stenosis model has a much larger plaque volume than its corresponding uniform stenosis model of a similar stenosis severity. WSS of the growth-updated stenosis model is elevated for both the diseased and healthy sides of the artery wall, accompanied with significant WSS fluctuations in the post-stenotic region. Pressure results also reveal a similar trend, where a larger pressure drop at the stenotic throat location is found when compared to the corresponding uniform stenosis model. All of these features suggest that a growth-updated stenosis model provides more realistic data for predicting plaque growth and rupture. Clinically, these results may provide some valuable assistance from a surgical point of view, as they highlight the increased risks of wall rupture on the healthy side of the artery at the stenosis region. Future work is focused on the inclusion of a lipid pool in various stenosis severity models 
with the possibility of a secondary plaque formation downstream and on the influence of intimal hyperplasia on plaque stability.

\section{References}

[1] C. X. Chen, Y. Ding and J. A. Gear, "Blood flow in stenosed arteries using two way, fluid-structural interaction", ANZIAM J. 51 (2010) C586-C611; http://journal.austms.org.au/ojs/index.php/ANZIAMJ/article/view/2575.

[2] X. Deng, Y. Marois, T. How, Y. Merhi, M. King and R. Guidoin, "Luminal surface concentration of lipoprotein (LDL) and its effects on the wall uptake of cholesterol by canine carotid arteries", J. Vasc. Surg. 21 (1995) 135-145; doi:10.1016/S0741-5214(95)70252-0.

[3] B. M. Johnston, P. R. Johnston, S. Corney and D. Kilpatrick, "Non-Newtonian blood flow in human right coronary arteries: steady state simulations", J. Biomech. 37 (2004) 709-720; doi:10.1016/j.jbiomech.2003.09.016.

[4] K. W. Lee and X. Y. Xu, "Modelling of flow and wall behaviour in a mildly stenosed tube", Med. Engrg. Phys. 24 (2002) 575-586; doi:10.1016/S1350-4533(02)00048-6.

[5] Z.-Y. Li and J. H. Gillard, "Simulation of the interaction between blood flow and atherosclerotic plaque", in: Proc. 29th Annual International Conference of the IEEE EMBS, 2007, 1699-1702; doi:10.1109/IEMBS.2007.4352636.

[6] T. Naiki and T. Karino, "Visualization of flow-dependent concentration polarization of macromolecules at the surface of a cultured endothelial cell monolayer by means of fluorescence microscopy", Biorheology 37 (2000) 371-384; http://iospress.metapress.com/content/29rx0j9dkkeuuyhj.

[7] M. Ojha, R. S. C. Cobbold, K. W. Johnston and R. L. Hummel, "Pulsatile flow through constricted tubes: an experimental investigation using photochromic tracer methods", J. Fluid Mech. 203 (1989) 173-197; doi:10.1017/S0022112089001424.

[8] U. Olgac, V. Kurtcuoglu and D. Poulikakos, "Computational modeling of coupled blood-wall mass transport of LDL: effects of local wall shear stress", Am. J. Physiol. Heart Circ. Physiol. 294 (2008) H909-H919; doi:10.1152/ajpheart.01082.2007.

[9] J. V. Soulis, T. M. Farmakis, G. D. Giannoglou and G. E. Louridas, "Wall shear stress in normal left coronary artery tree", J. Biomech. 39 (2006) 742-749; doi:10.1016/j.jbiomech.2004.12.026.

[10] J. V. Soulis, D. K. Fytanidis, V. C. Papaioannou and G. D. Giannoglou, "Wall shear stress on LDL accumulation in human RCAs", Med. Engrg. Phys. 32 (2010) 867-877;

doi:10.1016/j.medengphy.2010.05.011. 